\title{
Correspondence between Turning Points in Trend of Oil Price and Business Cycles in Japan
}

\author{
Koki Kyo ${ }^{1, a}$ and Hideo Noda ${ }^{2, b, *}$ \\ ${ }^{1}$ Obihiro University of Agriculture and Veterinary Medicine \\ Inada-cho, Obihiro, Hokkaido 080-8555, Japan \\ ${ }^{2}$ Tokyo University of Science \\ 1-11-2 Fujimi, Chiyoda-ku, Tokyo 102-0071, Japan \\ akyo@obihiro.ac.jp, bnoda@rs.tus.ac.jp \\ *Corresponding author
}

\begin{abstract}
Oil price is crucial to understanding economic fluctuations in Japan. In this paper, we decompose the time series of oil price in the period from January 1971 to December 2012 into a long-term tendency (also called trend) and shortterm variation, which is expressed by an autoregressive model. The correspondence between the turning points in the trend of oil price and business cycles in Japan is then analyzed. Particularly, after the 2000s, a clear relationship exists between turning points in the trend of real oil price and business cycles. Our results suggest that we can obtain useful information about Japanese business conditions by forecasting the trend of oil price.
\end{abstract}

Keywords-oil price; business cycle; turning points; Japanese economy; state space model

\section{INTRODUCTION}

Since the middle of the 1960s, Japan has depended on imports for more than 99 percent of its crude oil. Crude oil remains an important energy resource in the Japanese economy, and oil price fluctuations influence the economic activities of consumers and producers through various transmission mechanisms (see [1]). Oil price is thus a crucial factor in analyzing business fluctuations in the Japanese economy. This paper aims to understand the relationship between turning points in oil price trend and business cycles in Japan.

Reflecting strong interest from economists, policymakers, and financial market participants, many empirical studies have examined the influences of oil price fluctuations on the Japanese economy. Major studies in recent years include those of [2] and [3]. Hamilton analyzed data from the US economy to verify the hypothesis that an increase in oil price would have a negative effect on the macroeconomy but that a positive effect would not always result from a decrease in oil prices (Hamilton called this a nonlinear hypothesis). Zhang applied the approach of [2] to an analysis of the Japanese economy. The results in [3] suggest nonlinearity between changes in oil price and the growth rate of industrial production in Japan.

Notably, the models in [2] and [3] assume invariability in the coefficients of the explanatory variables. However, when we apply the time series modeling method over several decades, it is unrealistic to assume no structural change. To better capture patterns of structural change, Kyo and Noda [4] introduced a Bayesian dynamic regression modeling approach and a Bayesian time-varying coefficient vector autoregressive (TVCVAR) modeling approach. Accordingly, in [4] we found evidence from Japan that supported the existence of both structural changes and an increasing negative impact of oil price fluctuations on industrial production performance since the 2000s.

Some studies also focused on the stock market or cyclical unemployment. Using a structural vector autoregressive model, [5] examined the relationship between oil price shocks and the Japanese stock market. [5] found a positive correlation between oil price shocks arising from changes in aggregate global demand and stock market returns. That is, a rise in oil price is not always bad news for the Japanese stock market. However, the Japanese stock market reacts negatively to oil price increases related to oil market specific demand shocks. Applying a TVCVAR modeling approach, Kyo and Noda [6] also studied the influence of oil price fluctuations on business cycles and the unemployment rate in Japan. The results in [6] suggest that oil price fluctuations affect the cyclical unemployment rate during a recession. Additionally, oil price fluctuations recently have come to strongly influence business conditions and unemployment.

Earlier studies have obtained meaningful findings from various perspectives. However, no study has examined the relationship between turning points in oil price trend and business cycles in Japan. Therefore, we hope that this study can acquire important new findings.

Figure 1 shows the movement of oil price in the period from January 1971 to December 2012 against the vertical lines that correspond to the turning points of the business cycles in Japan. Looking at Figure 1, we find a possible relation between large changes in oil price and turning points of the business cycles. However, volatility complicates the behavior of oil price, making it difficult to clearly examine the correspondence between oil price variation and the business cycles in Japan. Thus, in this paper, we decompose the time series of oil price into a long-term tendency (called the trend) and short-term variations expressed by an autoregressive (AR) model. Then, we analyze the 
correspondence between turning points in the oil price trend and the business cycles in Japan.

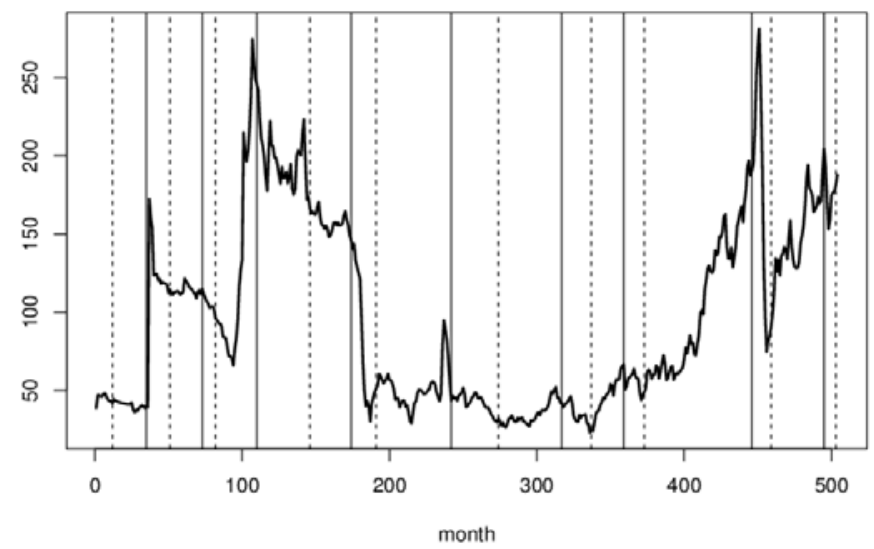

Figure 1. Real oil price with the vertical lines indicating turning points of the business cycle (monthly data in in the period Jan. 1971 Dec. 2012, the solid and broken lines indicate peaks and troughs, respectively); Sources: International Financial Statistics and Cabinet Office, Government of Japan.

The rest of this paper is organized as follows. In Section 2 , we present the model and estimation methods. Section 3 summarizes the main results and considers economic implications. Finally, Section 4 gives conclusions.

\section{Model And Estimation Method}

\section{A. Basic Model}

For monthly time series $y_{n}$ of oil price, we consider a set of statistical models as follows:

$$
\begin{aligned}
& y_{n}=t_{n}+r_{n}+w_{n}, \\
& t_{n}=2 t_{n-1}-t_{n-2}+v_{n 1}, \\
& r_{n}=\sum_{i=1}^{q} \alpha_{i} r_{n-i}+v_{n 2},
\end{aligned}
$$

where $t_{n}$ and $r_{n}$ are the trend component and the stationary AR component, respectively. Also, $q$ represents the order of the AR component model. $w_{n} \sim \mathrm{N}\left(0, \sigma^{2}\right)$ is the observation error, $v_{n 1} \sim \mathrm{N}\left(0, \tau_{1}^{2}\right)$ and $v_{n 2} \sim \mathrm{N}\left(0, \tau_{2}^{2}\right)$ are system noises. $w_{n}, v_{n 1}$ and $v_{n 2}$ are assumed to be independent of one another.

The models in (1) - (3) are essentially Bayesian linear models in which the model in (1) defines the likelihood and the models in (2) and (3) define the priors for $t_{n}$ and $r_{n}$, respectively. Especially, the model in (2) forms a second order smoothness prior for the trend $t_{n}$, so we can estimate the trend with very high smoothness. This model can be also considered a special form of the seasonal adjustment model in which a seasonal component is omitted and a stationary AR component is added (see [7] and [8] for detail of seasonal adjustment models). In this model, the trend component $t_{n}$ captures a long-term tendency, and the AR component $r_{n}$ expresses a short-term variation of the time series $y_{n}$.

In recent years, state space model approaches have proven very useful for analyzing complicated dynamic systems. This justifies expressing the models in (1) - (3) by the following state-space representation:

$$
x_{n}=F x_{n-1}+G_{n} v_{n}
$$

$$
y_{n}=H x_{n}+w_{n},
$$

where $x_{n}$ is the state vector with dimension $m=q+2, w_{n}$ is the observation noise vector, and $v_{n}$ is the system noise vector. The related vectors and matrices are defined, respectively, as follows:

$$
\begin{aligned}
& x_{n}=\left(t_{n}, t_{n-1}, r_{n}, \ldots, r_{n-q+1}\right)^{\mathrm{T}}, w_{n}=\left(w_{n}\right), v_{n}=\left(v_{n 1}, v_{n 2}\right)^{\mathrm{T}}, \\
& F=\left[\begin{array}{cccccc}
2 & -1 & 0 & \cdots & \cdots & 0 \\
1 & 0 & 0 & \cdots & \cdots & 0 \\
0 & 0 & \alpha_{1} & \alpha_{2} & \ldots & \alpha_{q} \\
0 & 0 & 1 & 0 & \ldots & 0 \\
\vdots & \vdots & \ddots & \ddots & \ddots & \vdots \\
0 & 0 & \cdots & 0 & 1 & 0
\end{array}\right], G=\left[\begin{array}{cc}
1 & 0 \\
0 & 0 \\
0 & 1 \\
0 & 0 \\
\vdots & \vdots \\
0 & 0
\end{array}\right], \\
& H=\left[\begin{array}{llllll}
1 & 0 & 1 & 0 & \cdots & 0
\end{array}\right] .
\end{aligned}
$$


Moreover, using the parameter transformation $d_{1}^{2}=\tau_{1}^{2} / \sigma^{2}, d_{2}^{2}=\tau_{2}^{2} / \sigma^{2}$ for a given value of $\sigma^{2}$ and letting $Q=E\left\{v_{n} v_{n}^{\mathrm{T}}\right\}=\operatorname{diag}\left(d_{1}^{2}, d_{2}^{2}\right), \quad R=E\left\{w_{n}^{2}\right\}=1$, then the parameters $q, \sigma^{2}, d_{1}^{2}, d_{2}^{2}, \alpha_{1}, \alpha_{2}, \ldots$, and $\alpha_{q}$ are used to define the above state space model and the state vector $x_{n}$ can be estimated using the Kalman filter algorithm, as shown below.

\section{B. Estimating the State Vector}

Let $x_{0}$ denote the initial state and $Y_{k}=\left\{y_{1}, y_{2}, \ldots, y_{k}\right\}$ denote a set of observations for $y_{n}$ up to the time point $k$. Assuming $x_{0} \sim \mathrm{N}\left(x_{0 \mid 0}, C_{0 \mid 0}\right)$, the distribution $f\left(x_{n} \mid Y_{k}\right)$ of the state $x_{n}$ conditional on $Y_{k}$ is well known to be Gaussian, so it is only necessary to obtain the mean $x_{n \mid k}$ and the covariance matrix $C_{n \mid k}$ of $x_{n}$ with respect to $f\left(x_{n} \mid Y_{k}\right)$.

Given the values of $q, \sigma^{2}, d_{1}^{2}, d_{2}^{2}, \alpha_{1}, \alpha_{2}, \ldots$, and $\alpha_{q}$, the distribution $\mathrm{N}\left(x_{0 \mid 0}, C_{0 \mid 0}\right)$ for $x_{0}$, and a set of observations for $y_{n}$ up to the time point $N$, then the estimates for the state $x_{n}$ can be obtained recursively using a well-known Kalman filter (for $n=1,2, \ldots, N$ ) and fixedinterval smoothing (for $n=N-1, N-2, \ldots, 1$ ) as follows (for example, see [9] and [10]):

[Kalman filter]

$$
\begin{aligned}
& x_{n \mid n-1}=F x_{n-1 \mid n-1}, \\
& C_{n \mid n-1}=F C_{n-1 \mid n-1} F^{\mathrm{T}}+G Q G^{\mathrm{T}}, \\
& L_{n}=C_{n \mid n-1} H^{\mathrm{T}}\left(H C_{n \mid n-1} H^{\mathrm{T}}+R\right)^{-1}, \\
& x_{n \mid n}=x_{n \mid n-1}+L_{n}\left(y_{n}-H x_{n \mid n-1}\right), \\
& C_{n \mid n}=\left(I-L_{n} H\right) C_{n \mid n-1} .
\end{aligned}
$$

[Fixed-interval smoothing]

$$
\begin{aligned}
& P_{n}=C_{n \mid n} F^{\mathrm{T}} C_{n+1 \mid n}^{-1}, \\
& x_{n \mid N}=x_{n \mid n}+P_{n}\left(x_{n+1 \mid N}-x_{n+1 \mid n}\right), \\
& C_{n \mid N}=C_{n \mid n}+P_{n}\left(C_{n+1 \mid N}-C_{n+1 \mid n}\right) P_{n}^{\mathrm{T}} .
\end{aligned}
$$

Here, $I$ denotes an identity matrix.

Then, the posterior distribution of $x_{n}$ can be defined using $x_{n \mid N}$ and $C_{n \mid N}$, and subsequently the trend $t_{n}$ and the AR component $r_{n}$ can be estimated because the state space model described by (4) and (5) incorporates $t_{n}$ and $r_{n}$ in the state vector $x_{n}$. Hereafter, the estimates of $t_{n}$ and $r_{n}$ are denoted by $\hat{t}_{n}$ and $\hat{r}_{n}$. Then, the estimated observation error $w_{n}$ is given by $\hat{w}_{n}=y_{n}-\hat{t}_{n}-\hat{r}_{n}$.

\section{Parameter Estimation}

When the time series data $Y_{N}=\left\{y_{1}, y_{2}, \ldots, y_{N}\right\}$ are given, a likelihood function for the parameters, $\sigma^{2}, \theta=\left\{d_{1}^{2}, d_{2}^{2}, \alpha_{1}, \alpha_{2}, \ldots, \alpha_{q}\right\}$ and $q$ is approximated by

$$
f\left(Y_{N} \mid \sigma^{2}, \theta, q\right)=\prod_{n=1}^{N} f_{n}\left(y_{n} \mid Y_{n-1} ; \sigma^{2}, \theta, q\right) \text {, }
$$

where $f_{n}\left(y_{n} \mid Y_{n-1} ; \sigma^{2}, \theta, q\right)$ is the conditional density function of $y_{n}$ given the history $Y_{n-1}=\left\{y_{1}, y_{2}, \ldots, y_{n-1}\right\}$. Assume that $Y_{0}$ is a empty set, then $f_{1}\left(y_{1} \mid Y_{0} ; \sigma^{2}, \theta, q\right)$ $=f_{1}\left(y_{1} \mid \sigma^{2}, \theta, q\right)$. By taking the logarithm of $f\left(Y_{N} \mid \sigma^{2}, \theta, q\right)$, the log-likelihood is obtained as

$$
\begin{aligned}
& \ell\left(\sigma^{2}, \theta, q\right)=\log f\left(Y_{N} \mid \sigma^{2}, \theta, q\right) \\
= & \sum_{n=1}^{N} \log f_{n}\left(y_{n} \mid Y_{n-1} ; \sigma^{2}, \theta, q\right) .
\end{aligned}
$$

As given by [10], using the Kalman filter the conditional density $f_{n}\left(y_{n} \mid Y_{n-1} ; \sigma^{2}, \theta, q\right)$ is a normal density given by

$$
\begin{array}{r}
f_{n}\left(y_{n} \mid Y_{n-1} ; \sigma^{2}, \theta, q\right)=\frac{1}{\sqrt{2 \pi \sigma^{2} u_{n \mid n-1}}} \\
\quad \times \exp \left\{-\frac{\left(y_{n}-\hat{y}_{n \mid n-1}\right)^{2}}{2 \pi \sigma^{2} u_{n \mid n-1}}\right\},
\end{array}
$$

where $\hat{y}_{n \mid n-1}$ is the one-step-ahead prediction for $y_{n}$ and $u_{n \mid n-1}$ is the variance of the predictive error, respectively given by

$$
\hat{y}_{n \mid n-1}=H x_{n \mid n-1}, \quad u_{n \mid n-1}=H C_{n \mid n-1} H^{\mathrm{T}}+R .
$$

Based on the above setting, the parameters can be estimated using the maximum likelihood method, and parameter estimation can be performed using the following three steps: First, for given values of $\theta$ and $q$, the estimate $\hat{\sigma}^{2}(\theta, q)$ for $\sigma^{2}$ is obtained analytically by:

$$
\hat{\sigma}^{2}(\theta, q)=\frac{1}{N} \sum_{n=1}^{N} \frac{\left(y_{n}-\hat{y}_{n \mid n-1}\right)^{2}}{u_{n \mid n-1}} .
$$



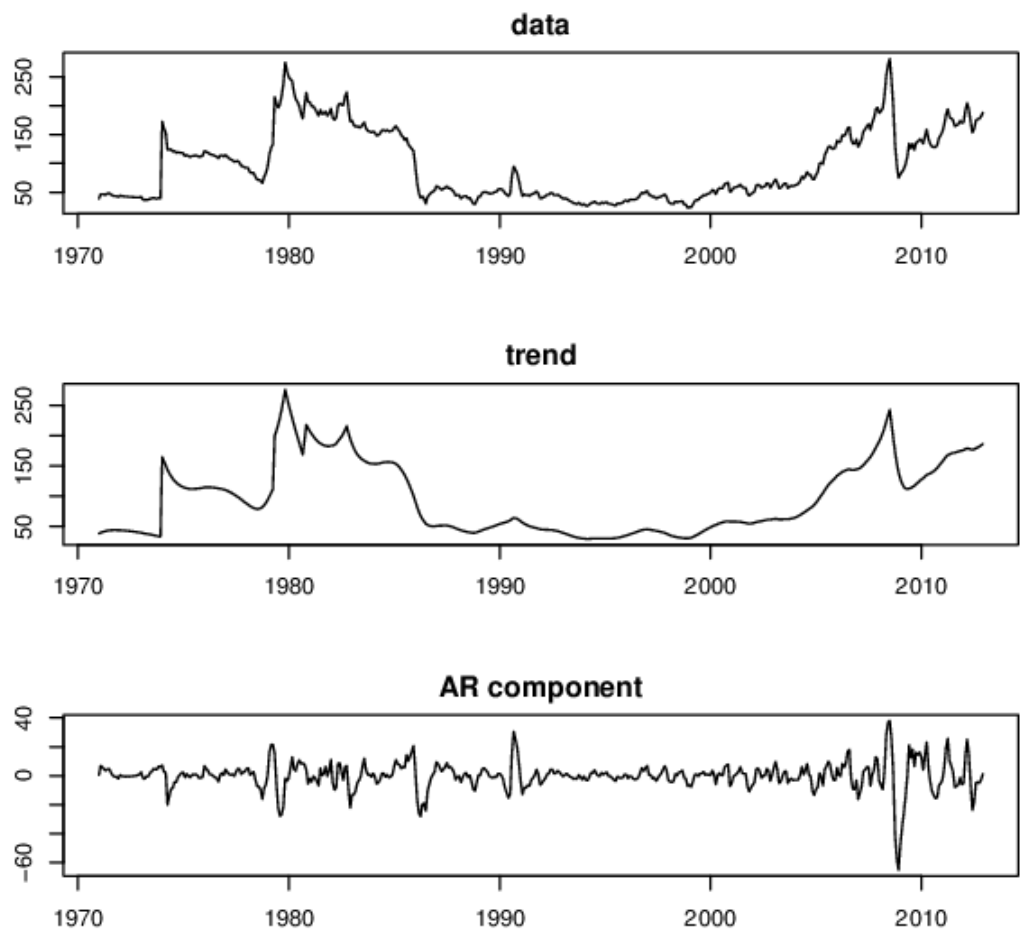

observation error

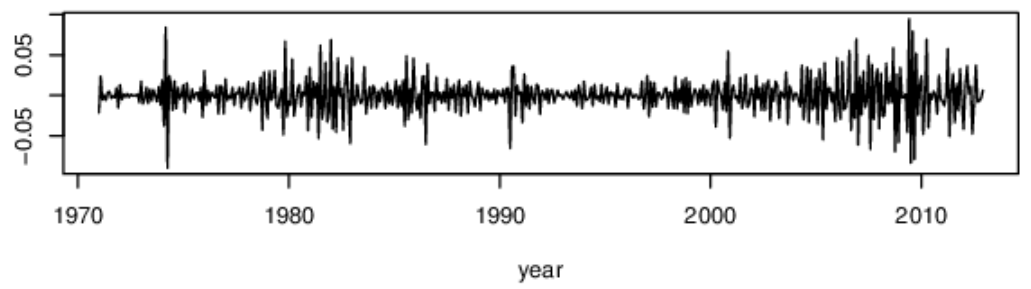

Figure 2. Real oil price and estimates of trend, AR component and observation error

Then, for a given value of $q$, the estimates of $\theta$ can be obtained numerically using maximum likelihood method; i.e., we can estimate $\theta$ by numerically maximizing $\ell\left(\hat{\sigma}^{2}(\theta, q), \theta, q\right)$ in (6) and (7). To save computational costs we used a recursive estimation method proposed by [8] to estimate the AR coefficients $\alpha_{1}, \alpha_{2}, \ldots, \alpha_{q}$. Finally, the value of $q$ is determined by the minimum Akaike information criterion (AIC) method (see [11]). Incidentally, the estimates of $\tau_{1}^{2}$ and $\tau_{2}^{2}$ are calculated from the estimates of $\sigma^{2}$, $d_{1}^{2}$ and $d_{2}^{2}$ by $\hat{\tau}_{1}^{2}=\hat{\sigma}^{2} \hat{d}_{1}^{2}, \hat{\tau}_{2}^{2}=\hat{\sigma}^{2} \hat{d}_{2}^{2}$.

\section{Searching for Turning Points in the Trend}

As mentioned, the main aim of this study is to understand the effects of oil price shocks on the Japanese economy by identifying the turning points distributed over the smooth trend of oil price. This necessitates estimating the trend using a non-Gaussian modeling method (see [12]). However, it is difficult for non-Gaussian modeling to estimate so many parameters.

Therefore, we search for turning points in the trend using the outlier detection method, as follows: First, we compute estimates for $v_{n 1}$ by $\hat{v}_{n 1}=\hat{t}_{n}-2 \hat{t}_{n-1}+\hat{t}_{n-2}$, and normalize the series $\hat{v}_{n 1}$ by $\hat{v}_{n 1}^{*}=\frac{\hat{v}_{n 1}}{\operatorname{sd}\left\{\hat{v}_{n 1}\right\}}$ with $\operatorname{sd}\left\{\hat{v}_{n 1}\right\}$ denoting the standard deviation of the time series $\hat{v}_{n 1}$. Next, we consider a value in the time series $\hat{v}_{n 1}$ as an outlier if $\hat{v}_{n 1}^{*}>2.5$ or $\hat{v}_{n 1}^{*}<-2.5$. This lets us replace the variances for the outliers in the time series $\hat{v}_{n 1}$ with a very large quantity. 


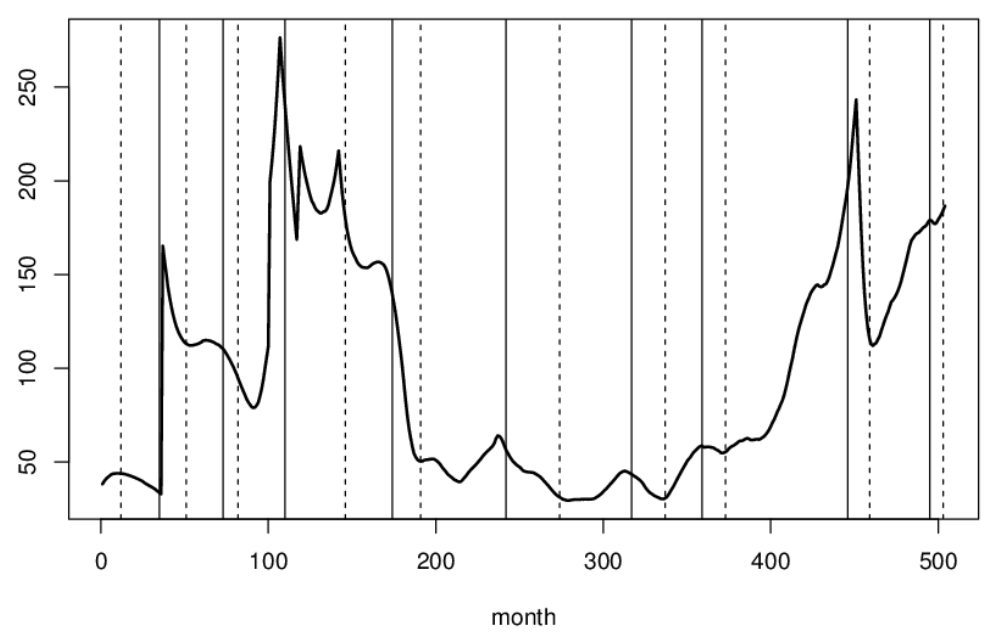

Figure 3. Estimated trend in the oil price time series with vertical lines indicating turning points of the business cycle (monthly data in in the period Jan. $1971 \sim$ Dec. 2012, solid and broken lines denote peaks and troughs, respectively).

\section{RESULTS AND IMPLICATIONS}

We applied the proposed approach to monthly oil price statistics from January 1971 to December 2012, as shown in Figure 1. Like the method described in [3], we also constructed time series data of real oil price in Japanese Yen (hereafter called real oil price), using the nominal exchange rate, Dubai spot price index, and consumer price index as data sources. These time series data are available from the database of International Financial Statistics.

Salient fluctuations in the graph of real oil price reflect major oil shocks since the 1970s, caused by the OPEC oil embargo of 1973-1974, the Iranian revolution of 1978-1979, the Iran-Iraq War that started in 1980, the first Persian Gulf War in 1990-1991, and the oil price spike of 2007-2008 (see [13] and [14], for details). Moreover, the solid and broken vertical lines in Figure 1 represent peaks and troughs, respectively, in business cycles in Japan. Regarding important reference dates for business cycles in Japan, the earliest broken line occurs in December 1971 and the latest broken line occurs in November 2012. The period between two troughs is considered to comprise a single business cycle. Japan has experienced 15 such cycles since the Second World War, and Figure 1 represents the seventh to the fifteenth of these.

Table 1 shows the AIC values for each value of the AR model order with $q=1,2, \ldots, 5$.

TABLE I. AIC VALUES FOR $q=1,2, \ldots, 5$.

\begin{tabular}{c|c|c|c|c}
\hline$q=1$ & $q=2$ & $q=3$ & $q=4$ & $q=5$ \\
\hline 3519.4 & 3482.2 & 3483.8 & 3485.3 & 3486.7 \\
\hline
\end{tabular}

From this table, we can see that the AIC value is minimized when $q=2$, so we determined the AR model order using $q=2$ based on the minimum AIC method. For this model, the estimates of parameters are as follows: $\quad \hat{\sigma}^{2}=0.086212, \quad \hat{\tau}_{1}^{2}=3.9047, \quad \hat{\tau}_{2}^{2}=36.1014$, $\hat{\alpha}_{1}=1.2853, \hat{\alpha}_{2}=-0.4282$.

Figure 2 shows four panels of the time series related to real oil price. The three panels at the top show the original data, estimated trend and estimated AR component, respectively, while the fourth panel shows the estimated observation error. Clearly, the trend of real oil prices exhibits a non-monotonic pattern. Furthermore, this pattern agrees with our intuition. That is, the trend indicates a rapid increase in the periods of oil shocks of 1973-1974, 1978-1979, and 2007-2008. Hence, we believe the trend can be extracted accurately. Focusing on the behavior of the AR component, we find the cyclical fluctuations of real oil prices to be irregular and unstable. Moreover, this instability has increased in recent years.

Here, we focus on the estimated trend of real oil price. Figure 3 shows this trend, and the vertical lines corresponding to turning points of the business cycle in Japan. As in Figure 1, the solid and broken lines in Figure 3 denote peaks and troughs in the business cycles in Japan, respectively. From Figure 3, we find the following characteristics. From the 1970s to the mid-1980s, two peaks of real oil price are located near lines that correspond to the turning points of November 1973 and February 1980. However, no clear relations can be found with other turning points in the business cycles during that period. For the period from the late 1980 s to the late 1990 s, a relation seems to exist between real oil price and turning points in the business cycles. Specifically, peaks in real oil prices lead turning points in the business cycles (February 1991 and May 1997). Finally, after the 2000s the relationship becomes stronger than it was from the late 1980s to the late 1990s. Additionally, in many cases the peaks and troughs in the trend of real oil price almost coincide with turning points in the business cycles during that period. Consequently, after 
the 2000s, the connection between real oil prices and the business cycles in Japan seems to strengthen. This is consistent with the main result in [4]: The effect of oil price fluctuations on industrial production has been increasing since the 2000s. Hence, it is interpreted that fluctuations in oil prices have exerted considerable influence on the Japanese business cycle even after the 2000s.

From the above observation, we can see a very strong correspondence between the turning points in the trend of real oil price and the business cycles in Japan. Particularly, the trend of oil price appears to be a crucial influence on Japanese business cycles after the 2000s. In other words, we can obtain useful leading information on business conditions in Japan by forecasting the trend of real oil price.

\section{CONCLUSION}

Because oil price fluctuations influence the economic activities of consumers and producers, we guess a possible relation between large changes in oil price and turning points of the business cycles. However, volatility complicates the behavior of oil price, making it difficult to clearly examine the correspondence between oil price variation and the business cycles in Japan.

In this paper, therefore, we decomposed the time series of real oil price in the period from January 1971 to December 2012 into a long-term trend and short-term variations using a state space modeling approach. The estimated trend of real oil price indicates a rapid increase in the periods of oil shocks of 1973-1974, 1978-1979, and 2007-2008. For the cyclical fluctuations of real oil price, we find a significantly higher instability in the period of oil shock of 2007-2008 compared to those of 1973-1974 and 1978-1979. Looking at the period after the 2000s, a clear relationship exists between turning points in the trend of real oil price and business cycles in Japan. Our results imply that the accurate forecasting for the trend of real oil price would be a helpful indicator of business conditions in Japan. Therefore, development of a method to predict the trend of oil price is an important issue for future research.

\section{ACKNOWLEDGMENT}

The authors wish to thank the anonymous reviewers for their constructive comments and suggestions, which have contributed to improving the readability and quality of this paper. This work is supported in part by a Grant-in-Aid for Scientific Research (C) (16K03591) from the Japan Society for the Promotion of Science.

\section{REFERENCES}

[1] C. Baumeister and L. Kilian, "Forty years of oil price fluctuations: why the price of oil may still surprise us," Journal of Economic Perspectives, Winter 2016, vol. 30, pp. 139-160, doi:10.1257/jep.30.1.139.

[2] J. D. Hamilton, "What is an oil shock?,” Journal of Econometrics, April 2003, vol. 113, pp. 363-398, doi:10.1016/S03044076(02)00207-5.

[3] D. Zhang, "Oil shock and economic growth in Japan: a nonlinear approach,” Energy Economics, vol. 30, September 2008, pp. 23742390, doi:10.1016/j.eneco.2008.01.006.

[4] K. Kyo and H. Noda, "Bayesian analysis of the dynamic relationship between oil price fluctuations and industrial production performance in Japan,” Information, vol. 16, July 2013, pp. 4639-4659.

[5] A. Abhyankar, B. Xu, and J. Wang, "Oil price shocks and the stock market: evidence from Japan,” The Energy Journal, vol. 34, April 2013, pp. 199-222, doi:10.5547/01956574.34.2.7.

[6] K. Kyo and H. Noda, "Dynamic effects of oil price fluctuations on business cycle and unemployment rate in Japan,” International Journal of Innovation, Management and Technology, vol. 6, December 2015, pp. 374-377, doi:10.18178/ijimt.2015.6.6.633.

[7] G. Kitagawa, Introduction to Time Series Modeling, New York: CRC Press, 2010.

[8] K. Kyo and H. Noda, "A new algorithm for estimating the parameters in seasonal adjustment models with a cyclical component, "ICIC Express Letters, vol. 5, May 2011, pp. 1731-1737.

[9] B. D. O. Anderson and J. B. Moore, Optimal Filtering, New Jersey: Prentice-Hall, 1979.

[10] G. Kitagawa and W. Gersch, Smoothness Priors Analysis of Times Series, New York: Springer-Verlag, 1996.

[11] H. Akaike, "A new look at the statistical model identification," IEEE Transactions on Automatic Control, vol. 19, December 1974, pp. 716723, doi:10.1109/TAC.1974.1100705.

[12] G. Kitagawa, "Monte carlo filter and smoother for non-Gaussian nonlinear state space models," Journal of Computational and Graphical Statistics, vol. 5, March 1996, pp. 1-25, doi:10.2307/1390750.

[13] S. Carollo, Understanding Oil Prices: A Guide to What Drives the Price of Oil in Today's Markets, New York: Wiley, 2012.

[14] J. D. Hamilton, "Historical oil shocks," in Routledge Handbook of Major Events in Economic History, R. E. Parker and R. Whaples, Eds. New York: Routledge Taylor and Francis Group, 2013, pp. 239-265. 